
\title{
25 Research Square \\ Erector Spinae Plane Block for Post-Operative Analgesia for Above the Knee Amputation: A Case Report
}

Erica Langnas ( $\nabla$ erica.langnas@ucsf.edu )

University of California San Francisco https://orcid.org/0000-0003-2902-8528

Andrew Gray

San Francisco General Hospital and Trauma Center: Zuckerberg San Francisco General Hospital and

Trauma Center

Matthias Braehler

UCSF: University of California San Francisco

\section{Case Study}

Keywords: regional anesthesia, erector spinae plane block, above the knee amputation, postoperative pain

Posted Date: August 3rd, 2021

DOI: https://doi.org/10.21203/rs.3.rs-754327/v1

License: (c) (i) This work is licensed under a Creative Commons Attribution 4.0 International License. Read Full License 


\section{Abstract}

Background: Above the knee amputations (AKA) are common surgeries that frequently use neuraxial or peripheral nerve blocking techniques for both intraoperative and postoperative analgesia. It is not uncommon that patients present with contraindications to neuraxial anesthesia.

Case presentation: We identified a relatively novel use of erector spinae plane block (ESP) for above the knee amputation that allows for adequate pain control postoperatively when there are contraindications for neuraxial.

Conclusion: While data on ESP at the thoracic level is well described, less is known about the expected coverage for lumbar ESP. This case suggests that at the level of L3 there is sufficient dermatomal spread for an AKA.

\section{Introduction:}

Above the knee amputations (AKA) are common surgeries that frequently use neuraxial or peripheral nerve blocking techniques for both intraoperative and postoperative analgesia. We aim to add to the limited existing literature that erector spinae plane blocks (ESP) may be utilized for above the knee amputation to reduce postoperative pain in patients who may have contraindications to neuraxial or peripheral nerve blocks. ${ }^{1-2}$ ESP blocks are relatively novel and have been gaining popularity and proving effective for postoperative pain for many surgeries. ${ }^{3-6}$ This case adds clinical evidence to the limited existing literature surrounding expectations of craniocaudal spread. ${ }^{6}$

Common regional anesthesia considerations for AKA are epidural, lumbar plexus, and peripheral nerve blocks. Epidural catheters may be used for surgical anesthesia and continued in the postoperative period. Limitations of epidurals are largely surrounding inability to safely place them. Contraindications include patients with coagulopathy, receiving anticoagulation, and infection at insertion site. Lumbar plexus blocks may be used for intra- and postoperative pain control. Limitations of this block are similar to those for an epidural catheter in regard to anticoagulation given the depth of the block as well as its proximity to central neural structures. ${ }^{7}$ Femoral nerve block or fascia iliaca block alone or in conjunction with sciatic nerve block may be used for post-operative pain control. Limitations include potential sparing of the obturator nerve, resulting in inferior pain control. The patient provided written HIPAA authorization for details of the case to be published.

\section{Case Presentation:}

We describe the case of a 74-year-old man who was scheduled for an urgent AKA. The patient was urgently admitted from home after his home nurse noticed dehiscence of his prior right below the knee amputation (BKA) stump. He received his BKA surgery one month prior for critical limb ischemia due to vascular disease which was complicated by poor wound healing. During this prior admission for a BKA 
he also received a right sided common femoral endarterectomy procedure. His past medical history is significant for end stage renal disease status post transplantation, aortic stenosis status post transcatheter aortic valve replacement, and atrial fibrillation for which he is on apixaban, heart failure with reduced ejection fraction and peripheral vascular disease. Due to the concern for wound dehiscence and infection noted by his home nurse, he was admitted urgently, started on broad spectrum antibiotics and scheduled for an AKA. Given the likelihood of surgery, his apixaban was held on admission and he was transitioned to a heparin drip.

On the morning of surgery his heparin drip was stopped four hours prior to surgery and a PTT was measured pre-operatively which returned normal. Given the need for anticoagulation after the surgery and concerns for difficulty of placement given patient body habitus with BMI of 41 , neuraxial was avoided and the anesthesia team planned to proceed with a peripheral nerve block. At the request of the surgeons, there would be no catheter placement. In preparation for the femoral nerve block, it was noted that the site of his prior right common femoral endarterectomy appeared erythematous and concerning for soft tissue infection. As a result, the femoral nerve block was aborted. Given these limitations, it was decided to place a right sided erector spinae plane block in the lumbar region. The patient was then positioned on his left side and a preliminary scan was completed at the third lumbar space (L3). This level was chosen for two reasons. First, we expected spread one to two levels above and below along and second, at this level we encountered the best ultrasound image quality. Midline, iliac crest, and the right transverse process were identified at L3. Preparation of the field with sterile preparation was completed. A 10 millimeter 21-gauge needle was inserted under ultrasound guidance. Gentle contact was made with the transverse process. Aspiration was negative and 30 milliliters of $0.2 \%$ ropivacaine was injected under direct visualization. Local anesthetic spread was noted below the erector spinae muscle.

Intraoperatively the patient received general anesthesia. Induction medications included propofol, fentanyl, rocuronium and lidocaine. General anesthesia was maintained with sevoflurane and he received a total of $1 \mathrm{mg}$ of hydromorphone in incremental doses over the last hour of the case. He was extubated and taken to the post-anesthesia care unit without complications.

While in the PACU the patient's pain was monitored using a numerical rating scale from 0 to $10(0=$ no pain, 10 = worst imaginable pain). His first three documented pain score levels were zero and over the next three hours increased to a maximum pain score of 4 for which he received $1 \mathrm{gram}$ of acetaminophen and 5 milligrams of oxycodone. He recovered appropriately and was transferred to the ward. The patient reported pain scores of 0 to 4 for 11 hours postoperatively. On hour 12 his surgical site pain increased to 7 , requiring use of oxycodone and hydromorphone. We hypothesize this change in pain occurred as the nerve block wore off. On post-operative day one, the patient was interviewed about his experience with the nerve block. Consistent with the pain scores documented, he reported adequate pain control for about 10-12 hours and then an abrupt change as the nerve block wore off.

\section{Discussion:}


It is not uncommon that patients present with contraindications to neuraxial anesthesia. Here we identified a relatively novel use of ESP for above the knee amputation that allows for adequate pain control postoperatively. While data on ESP at the thoracic level is well described, less is known about the expected coverage for lumbar ESP. This case suggests that at the level of $L 3$ there is sufficient dermatomal spread for an AKA.

Compared to femoral nerve block and fascia iliaca block, which do not necessarily cover the obturator nerve, the lumbar ESPB causes spread of local anesthetic to the roots of the lumbar plexus, which includes the obturator nerve. This is critical as the obturator nerve innervates a major part of the femur which contributes to postoperative pain. ${ }^{8}$ While a lumbar plexus block could be performed, there are concerns regarding anticoagulation given the depth of the block and its close proximity to neuraxial structures. The lumbar ESPB, however, is considered a more superficial block and there is emerging literature deeming it safe to perform this block using less strict guidelines compared with neuraxial and lumbar plexus blocks. ${ }^{9}$

Beyond the perioperative period, AKA surgery is associated with post amputation pain or phantom limb pain. ${ }^{10}$ This type of pain results in significant morbidity and remains challenging to treat. The etiology is complex and strategies to prevent or reduce the likelihood of post amputation continue to be explored. It is unknown if lumbar ESP catheters reduce post amputation pain and this may represent a novel area for future studies. In addition, we described in our case discomfort from the surgical team in placing a catheter given anticoagulation requirements for the patient. When new blocks emerge, there may be discomfort with surgical teams surrounding catheter placement. We encourage dialogue with surgical teams surrounding current literature and guidelines and the potential benefits of ESP blocks and how they compare with other common regional or neuraxial techniques.

In summary, this case report adds to relatively limited existing literature surrounding the potential uses of a lumbar ESP block. It is critical to increase the body of evidence on ESP blocks for orthopedic surgeries as this may result in more rigorous studies that compare effectiveness of ESP with other blocks.

\section{Abbreviations}

AKA

above the knee amputation

ESP

erector spinae plane

BKA

below the knee amputation

L3

third lumbar space

\section{Declarations}


Ethics approval and consent to participate: The patient provided written HIPAA authorization for details of the case to be published. Case reports are not required to undergo IRB review at our institution.

Availability of data and materials: Data sharing is not applicable to this article as no datasets were generated or analyzed during the current study.

Competing interests: The authors confirm they have no competing interests.

Funding: None to disclose.

Acknowledgements: None to disclose.

\section{Author Contributions:}

Erica Langnas is first author as she performed literature review, data collection and interpretation, and drafted the manuscript.

Andrew Gray provided critical revision of the manuscript for intellectual content and provided input on the concept and design of this paper.

Matthias Braehler provided critical revision of the manuscript for intellectual content and provided input on the concept and design of this paper.

Conflicts of Interests/Financial Disclosures: NONE

\section{References}

1. Ayub A, Talawar P, Gupta SK, Kumar R, Alam A. Erector spinae plane block: A safe, simple and effective alternative for knee surgery. Anaesth Intensive Care. 2019;47(5):469-71. doi:10.1177/0310057X19877655.

2. Balaban 0 , Aydın T. Lumbar erector spinae plane catheterization for continuous postoperative analgesia in total knee arthroplasty: A case report. J Clin Anesth. 2019;55:138-9. doi:10.1016/j.jclinane.2018.12.01.

3. Forero M, Adhikary SD, Lopez $\mathrm{H}$, et al. The erector spinae plane block: a novel analgesic technique in thoracic neuropathic pain. Reg Anesth Pain Med. 2016;41:621-7.

4. Chin KJ, Malhas L, Perlas A. The erector spinae plane block provides visceral abdominal analgesia in bariatric surgery: a report of 3 cases. Reg Anesth Pain Med. 2017;42:372-6.

5. Tulgar S, Senturk O. Ultrasound guided erector spinae plane block at L-4 transverse process level provides effective postoperative analgesia for total hip arthroplasty. J Clin Anesth. 2018;44:68.

6. Harbell MW, Seamans DP, Koyyalamudi V, Kraus MB, Craner RC, Langley NR. Evaluating the extent of lumbar erector spinae plane block: an anatomical study. Reg Anesth Pain Med. 2020 Aug;45(8):6404. PMID: 32546551. 
7. Horlocker TT, Vandermeuelen E, Kopp SL, Gogarten W, Leffert LR, Benzon HT. Regional Anesthesia in the Patient Receiving Antithrombotic or Thrombolytic Therapy: American Society of Regional Anesthesia and Pain Medicine Evidence-Based Guidelines (Fourth Edition). Reg Anesth Pain Med. 2018 Apr;43(3):263-309. PMID: 29561531.

8. Gadsden J, Warlick A. Regional anesthesia for the trauma patient: improving patient outcomes. Local Reg Anesth. 2015;8:45-55. doi:10.2147/LRA.S55322. Published 2015 Aug 12.

9. Adhikary SD, Prasad A, Soleimani B, Chin KJ. Continuous Erector Spinae Plane Block as an Effective Analgesic Option in Anticoagulated Patients After Left Ventricular Assist Device Implantation: A Case Series. J Cardiothorac Vasc Anesth. 2019 Apr;33(4):1063-7. PMID: 29753668.

10. Subramaniam B, Pomposelli F, Talmor D, Park KW. Perioperative and long-term morbidity and mortality after above-knee and below-knee amputations in diabetics and nondiabetics. Anesth Analg. 2005 May;100(5):1241-7. PMID: 15845661. 